\title{
Correction to: Effects of society and culture on parents' ratings of children's mental health problems in 45 societies
}

\author{
Leslie A. Rescorla ${ }^{1} \cdot$ Robert R. Althoff ${ }^{2} \cdot$ Thomas M. Achenbach $^{2} \cdot$ Masha Y. Ivanova $^{2} \cdot$ International ASEBA \\ Consortium
}

Published online: 12 March 2019

○) Springer-Verlag GmbH Germany, part of Springer Nature 2019

\section{Correction to: European Child \& Adolescent Psychiatry https://doi.org/10.1007/s00787-018-01268-3}

Unfortunately, due to a technical error the International ASEBA Consortium was not listed as author in the original publication. This error is corrected via this correction.

All members of the consortium are listed in the acknowledgments.

Acknowledgments Drs. Rescorla, Achenbach, and Ivanova receive support from the nonprofit Research Center for Children, Youth, and Families, which publishes the Child Behavior Checklist. Dr. Althoff receives grant or research support from the National Institute of Mental Health (MH082116), National Institute of Health Center of Biomedical Research Excellence award (P20GM103644), and Klingenstein Third Generation Foundation. Members of the ASEBA International Consortium who generously provided data for this paper include: Fredrik Almqvist (Finland), Ivan Begovac (Croatia), Niels Bilenberg (Denmark), Hector Bird (Puerto Rico), Myriam Chahed (Tunisia), Wei Chen (Taiwan), Dang Hoang-Minh (Vietnam), Anca Dobrean (Romania), Manfred Döpfner (Germany), Nese Erol (Turkey), Eric Fombonne (France), Antonio Fonseca (Portugal), Alessandra Frigerio (Italy), Hans Grietens (Belgium), Mariam Haider (Pakistan), Helga Hannesdottir (Iceland), Sri Hartini (Indonesia), , Walter Hellinckx (Russia), Nohelia Hewitt Ramirez (Colombia), Yasuko Kanbayashi (Japan), Hans Koot (Kenya), Michael Lambert (Jamaica), Bo Larsson (Sweden), Patrick Leung (Hong Kong), Xianchen Liu (China), Dorcas Magai

The original article can be found online at https://doi.org/10.1007/ s00787-018-01268-3.

\footnotetext{
Leslie A. Rescorla

1rescorl@brynmawr.edu

Bryn Mawr College, Bryn Mawr, USA

2 University of Vermont, Burlington, USA
}

(Kenya), Jasminka Markovic (Serbia), Asghar Minaei (Iran), Marina Monzani da Rocha (Brazil), Mesfin Mulatu (Ethiopia), Torunn Novik (Norway), Kyung-Ja Oh (South Korea), Dajouida Petot (Algeria), Jean-Michel Petot (Algeria), Julia Plück (Germany), Rolando Pomalima (Peru), Alexandra Roussos (Greece), Vlasta Rudan (Croatia), Michael Sawyer (Australia), Mimoza Shahini (Kosovo), Edwiges Silvares (Brazil), Zeynep Simsek (Turkey), Hans-Christoph Steinhausen (Switzerland), Jose Valverde (Peru), Frank Verhulst (The Netherlands), Laura Viola (Uruguay), Bahr Weiss (Vietnam), John Weisz (Thailand), Christa Winkler Metzke (Switzerland), Tomasz Wolanczyk (Poland), Bernardine Woo (Singapore), Hao-Jan Yang (Taiwan), Nelly Zilber (Israel), and Rita Zukauskiene (Lithuania). 